\title{
Einstein gravity of a diffusing fluid
}

\author{
Z. Haba \\ Institute of Theoretical Physics, University of Wroclaw, \\ 50-204 Wroclaw, Plac Maxa Borna 9, Poland \\ email:zhab@ift.uni.wroc.pl
}

August 13, 2018

\begin{abstract}
We discuss Einstein gravity for a fluid consisting of particles interacting with an environment of some other particles. The environment is described by a time-dependent cosmological term which is compensating the lack of the conservation law of the energy-momentum of the dissipative fluid. The dissipation is approximated by a relativistic diffusion in the phase space. We are interested in a homogeneous isotropic flat expanding Universe described by a scale factor $a$. At an early stage the particles are massless. We obtain explicit solutions of the diffusion equation for a fluid of massless particles at finite temperature. The solution is of the form of a modified Jüttner distribution with a time-dependent temperature. At later time Universe evolution is described as a diffusion at zero temperature with no equilibration. We find solutions of the diffusion equation at zero temperature which can be treated as a continuation to a later time of the finite temperature solutions describing an early stage of the Universe. The energy-momentum of the diffusing particles is defined by their phase space distribution. A conservation of the total energy-momentum determines the cosmological term up to a constant. The resulting energymomentum inserted into Einstein equations gives a modified Friedmann equation. Solutions of the Friedmann equation depend on the initial value of the cosmological term. The large value of the cosmological constant implies an exponential expansion. If the initial conditions allow a power-like solution for a large time then it must be of the form $a \simeq \tau$ (no deceleration, $\tau$ is the cosmic time). The final stage of the Universe evolution is described by a non-relativistic diffusion of a cold dust.
\end{abstract}

\section{Introduction}

We consider the gravitational Einstein equations in the form

$$
R^{\mu \nu}-\frac{1}{2} h^{\mu \nu} R=8 \pi G T^{\mu \nu},
$$


where $G$ is the Newton constant, $h^{\mu \nu}$ is the metric and the Einstein tensor on the lhs is covariantly conserved. Hence,

$$
\left(T^{\mu \nu}\right)_{; \mu}=0 .
$$

We could insert on the rhs of eq.(1) the energy-momentum $T^{\mu \nu}$ of a collection of particles described by a probability distribution $\Phi$ on the phase space [1][2][3][4]. If particle's dynamics is determined by classical evolution equations, then the conservation law (2) is a consequence of the Liouville equation (where $\Gamma_{\nu \rho}^{\mu}$ are Christoffel symbols)

$$
\left(p^{\mu} \partial_{\mu}^{x}-\Gamma_{\mu \nu}^{k} p^{\mu} p^{\nu} \partial_{k}\right) \Phi=0
$$

Eqs.(1),(3) and the formula for the energy-momentum tensor

$$
T^{\mu \nu}=\sqrt{h} \int \frac{d \mathbf{p}}{(2 \pi)^{3}} \frac{1}{p_{0}} p^{\mu} p^{\nu} \Phi,
$$

define the Einstein-Vlasov system [4]. In eq.(4) $h$ is the determinant of the metric and $p_{0}$ is determined from the mass-shell condition $p_{\mu} p^{\mu}=m^{2}$ ( $m$ is the particle's mass, we set $c=1$ ). In eqs.(1)-(4) Greek indices run from 0 to 3 , Latin indices denoting spatial components have the range from 1 to 3 , the covariant derivative is over the space-time, derivatives over the momenta $\frac{\partial}{\partial p^{k}}$ are denoted $\partial_{k}$ and $\partial^{x}$ denotes a derivative over a space-time coordinate $x$.

The Einstein-Vlasov system has been successfully applied [4] in a description of the large scale structure of the Universe. The deterministic approach (1)-(4) must be modified if we describe only a part of the total system. In such a case we do not have the complete information, e.g., we do not detect some forms of matter. The unknown part of the total system is excluded from a deterministic description. The system's motion is not deterministic but rather random. Under the assumption of a short memory (Markov approximation) the random motion can be described as a diffusion. The non-relativistic diffusion approximation has been widely applied in many body systems [5][6]. We consider a relativistic diffusion of the hot matter beginning from the early time of the Universe expansion. We apply the diffusion approximation to the description of motion of the luminous matter in the Universe assuming that this matter is formed from known elementary particles (there is an alternative approach describing the dark matter evolution as a diffusion [10]; for a discussion of the dark matter phase space distribution see [11]). We suggest that there are some other particles and interactions which we do not know. Their impact is described as a diffusive motion of matter. The gravitational attraction by distant objects as a source of the diffusive motion of stars has been described in [7] and confirmed by observations [8] [9]. In this paper the diffusion approximation is applied to all stages of the evolution. The energy-momentum tensor $T_{\mu \nu}$ on the rhs of eq.(1) consists of two parts: the first part expressed in eq.(4) (which is not conserved if $\Phi$ satisfies a diffusion equation) and the second part corresponding 
to the unknown component of the total system which is to be determined from the conservation law (2).

We could consider a Big Bang theory of a fluid consisting of particles of the standard grand unification model [12][13]. The heavy ion collisions could supply experiments verifying some assumptions of the Big Bang theory starting from microscopic kinetic theory [13][14][15]. We are unable to treat the formation of hadrons from quarks, decay and creation of particles without a complete quantum theory. We rely on a classical approximation of quantum theories. We approximate interactions by a diffusion. We have studied such an approximation in [16] where an interaction of relativistic charged particles with the $\mathrm{CMB}$ radiation is treated as a diffusion model. In the same way we can show that the quark-gluon interaction leads to a diffusion of quarks [17]. Even if the interactions are unknown the relativistic invariance determines to a large extent the form of the diffusion. The relativistic diffusion has been discovered in [18] and [19]. A model with friction leading to the Jüttner equilibrium distribution [20] is discussed in [21] [22][23][24]. An equilibration to a quantum distribution requires a non-linear diffusion [25] which is to describe the bunching of Bose particles and repulsion of Fermi particles. We treat the linear diffusion as an approximation to the non-linear one. We think that the relativistic diffusion can be a useful approximation for dissipative processes in the description of the early Universe. The non-relativistic diffusion as an approximation for the collision term in the Boltzmann equation has been elaborated in many papers (see [5][6][9]; for a recent application in cosmology see [3] and references cited there). An equation for the diffusion of photon energy in a medium of free non-relativistic electrons is well-known as the Kompaneets equation [26][27]. A relativistic diffusion approximation for the Boltzmann equation is discussed in [28].

In this paper we are going to elaborate the scheme suggesting that some unknown interactions are approximated by a relativistic diffusion. We assume that the particles are massless (we discuss a diffusion of massless particles in [25] [29][30]). In the grand unified theories elementary particles acquire a mass through a symmetry breaking at lower temperature. Hence, the zero mass assumption at an early time (the radiation era) is justified. The CMB radiation [31] shows the quantum origin of the (Planck) equilibrium distribution. We obtain a solution of the non-linear diffusion equation in the form of the Planck distribution with a time dependent temperature. In the ultrarelativistic limit and low density the non-linear diffusion is approximated by the linear diffusion. We solve the linear relativistic diffusion equation exactly. We find initial states of the Jüttner type [20] whose form is preserved by the time evolution. Such (steady) states are analogs of the equilibrium states in an expanding Universe. Moreover, they can be considered as asymptotic phase space distributions in the sense that any solution of the diffusion equation is approaching the Jüttner distribution with a time-dependent temperature. In a later time of the Universe evolution (lepton era) when other kinds of matter appear with a different local 
temperature we do not expect that the phase space distribution is approaching the equilibrium distribution. We describe this later stage of time evolution of the energy-momentum by a diffusive fluid of zero temperature (no heat bath).In order to perform exact calculations we still apply an ultrarelativistic approximation setting the mass of particles equal to zero . The diffusion constant is defined by the cross section of particle's interactions [28]. It can vary depending on the content of the system. We assume that this variation is continuous so that we obtain a continuous transition from the description in different stages of the Universe evolution (we denote the diffusion constant at various stages of the Universe expansion by the same symbol). The relativistic diffusion at zero temperature with no equilibrium distribution has been studied by Schay [18] and Dudley[19]. It has been applied for a description of a cosmological evolution of a diffusing fluid at a later stage of the expansion in [32] and [33]. In this paper we discuss exact solutions of the diffusion equation in the massless limit applied to early stages of the evolution. We obtain a continuation of the time-dependent Jüttner distribution at an early time to the solution of the diffusion equation at zero temperature at later time. We insert the energy-momentum corresponding to the solutions of the diffusion equation into the Einstein equations (1)

$$
R^{\mu \nu}-\frac{1}{2} h^{\mu \nu} R=T^{\mu \nu}=T_{D}^{\mu \nu}+\tilde{T}^{\mu \nu}
$$

where $T_{D}$ is the energy-momentum of a certain (dark) matter and $\tilde{T}$ is the energy-momentum of the system of diffusing particles. From the lhs it follows that

$$
\left(T_{D}^{\mu \nu}\right)_{; \mu}=-\left(\tilde{T}^{\mu \nu}\right)_{; \mu} .
$$

Knowing the rhs of eq.(6) we can determine the lhs up to a constant. We represent $T_{D}$ by a time-dependent cosmological term $\Lambda$. The cosmological term $\Lambda$ acquires a dynamical content in a way similar to the trace free formulation of Einstein equations [34][35]. A dynamical relation of the cosmological term to the matter density seems to be unavoidable for an explanation of the coincidence problem [36] [37]. In contradistinction to the Einstein-Vlasov system the dissipative system (5) gives a relation which could answer the question why the dark matter and "barionic" matter are of the same order of magnitude. We show that such a cosmological term is decreasing in time starting from a constant at a fixed time $\tau_{0}$ (which can be chosen as the radiation decoupling time). After an addition of the cosmological term $T_{D}$ the total energy-momentum $T$ can violate the strong energy condition as well as the dominant energy condition [38]. In the case of a homogeneous isotropic Universe we obtain an analog of the Friedmann equation for the scale factor $a$. The solutions of the modified Friedmann equations depend on the value of the cosmological constant. They can grow exponentially. We put the particle's mass equal to zero in early stages of the evolution. At the final stage of the expansion, when matter has already a macroscopic form, we consider a non-relativistic approximation (a large mass 
limit) of the diffusion equation. We discuss the resulting Friedmann equation for the scale factor $a$. We find an exact solution of the non-relativistic diffusion equation starting from the Maxwell equilibrium distribution. We insert the corresponding energy-momentum tensor in Einstein equations. The qualitative results for $a$ in the non-relativistic limit are similar to those of the relativistic massless case.

The plan of the paper is the following. In sec.2 we review the relativistic diffusion. In sec. 3 we derive solutions of the relativistic diffusion equation for massless particles at finite temperature. In sec. 4 we consider the limit of zero temperature of the solutions of sec.3. We discuss the energy-momentum tensor on the rhs of Einstein equations in sec.5. In sec.6 we obtain some consequences of the Friedmann equation resulting from Einstein equations with a diffusing fluid. A model of non-relativistic diffusing particles of a large mass (a cold "dust") is discussed in sec.7. The final section contains a summary and an outlook.

\section{Relativistic diffusion}

The diffusion is usually treated as an approximation for the collision term in the kinetic equation [5]. The diffusion approximation for the relativistic kinetic equation [39] has not been developed in a sufficient detail (see however [28]). If we look for a relativistic generalization of the Kramers diffusion defined on the phase space then it is determined in the unique way by the requirement that the diffusing particle moves on the mass-shell $\mathcal{H}_{+}($see $[18][19][21][22][30][40])$

$$
h_{\mu \nu} p^{\mu} p^{\nu}=m^{2}
$$

with the metric (flat expanding Universe)

$$
d s^{2}=h_{\mu \nu} d x^{\mu} d x^{\nu}=d \tau^{2}-\delta_{j k} a(\tau)^{2} d x^{j} d x^{k}=a^{2}(\tau(t))\left(d t^{2}-d \mathbf{x}^{2}\right),
$$

where

$$
t=\int d \tau(a(\tau))^{-1}
$$

is the conformal time (we call $\tau$ the cosmic time; the notation follows the textbook of Durrer [41]).

The diffusion is generated by the Laplace-Beltrami operator on $\mathcal{H}_{+}$

$$
\triangle_{H}=\frac{1}{\sqrt{g}} \partial_{j} g^{j k} \sqrt{g} \partial_{k}
$$

where

$$
g^{j k}=m^{2} h^{j k}+p^{j} p^{k}
$$


$\partial_{j}=\frac{\partial}{\partial p^{j}}$ and $g=\operatorname{det}\left(g_{j k}\right)$ is the determinant of $g_{j k}$. The operator $\triangle_{H}$ can be expressed in the form

$$
\triangle_{H}=m^{2} a^{-2} \delta^{j k} \partial_{j} \partial_{k}+p^{j} p^{k} \partial_{j} \partial_{k}+3 p^{k} \partial_{k},
$$

where $k=1,2,3$.

The transport equation for the linear diffusion generated by $\triangle_{H}$ reads

$$
\left(p^{\mu} \partial_{\mu}^{x}-\Gamma_{\mu \nu}^{k} p^{\mu} p^{\nu} \partial_{k}\right) \Omega=\kappa^{2} \triangle_{H} \Omega
$$

where $\kappa^{2}$ is the diffusion constant, $\partial_{\mu}^{x}=\frac{\partial}{\partial x^{\mu}}$ and $x=(t, \mathbf{x})$ (boldface letters denoting the three vectors).

The relativistic diffusion as an approximation of the relativistic Boltzmann equation [39] is discussed in ref.[28]. It is shown in [28] that the diffusion constant $\kappa^{2}$ is proportional to the cross section describing a scattering by particles of the medium . The diffusion matrix $g^{j k}$ is expressed by some integrals which have not been calculated explicitly in [28].

Eqs.(9)-(10) and (12) hold true for a general metric $h_{\mu \nu}$ in the gauge $h_{0 j}=0$ ( for a coordinate independent formulation see [42]). The specific realization of eq.(12) depends on the use of either lower case [30][32][33][40], upper case [42] or physical momenta $(\bar{p}=a p)[43]$. In this paper we use the upper case momenta ( the formula $p^{j}=h^{j k} p_{k}$ relates various formulations).

We consider also a diffusion at finite temperature $\beta^{-1}[21][22]$ (in the conformal time and in a frame moving with the heat bath)

$$
\left(p^{\mu} \partial_{\mu}^{x}-\Gamma_{\mu \nu}^{k} p^{\mu} p^{\nu} \partial_{k}\right) \Omega=\kappa^{2} p^{0} \partial_{j}\left(g^{j k} \frac{1}{p^{0}} \partial_{k}+\beta a^{2} p^{j}\right) \Omega .
$$

Here, $p^{0}$ is determined from eq.(7). So, in the conformal time

$$
p^{0}=\sqrt{a^{-2} m^{2}+\mathbf{p}^{2}}
$$

where $\mathbf{p}^{2}=\sum_{j} p^{j} p^{j}$

In the metric (8) the non-vanishing Christoffel symbols are

$$
\begin{aligned}
\Gamma_{j k}^{0} & =a^{-1} \frac{d a}{d t} \delta_{j k} \\
\Gamma_{0 k}^{j} & =a^{-1} \frac{d a}{d t} \delta_{k}^{j} .
\end{aligned}
$$

For rotation invariant and space homogeneous $\Omega$ eq.(12) reads

$$
\begin{aligned}
& \sqrt{p^{2}+a^{-2} m^{2}}\left(\partial_{t} \Omega-2 \mathcal{H} p \frac{\partial \Omega}{\partial p}\right) \\
& =\kappa^{2}\left(a^{-2} m^{2} \frac{\partial^{2} \Omega}{\partial p^{2}}+2 a^{-2} m^{2} p^{-1} \frac{\partial \Omega}{\partial p}+p^{2} \frac{\partial^{2} \Omega}{\partial p^{2}}+3 p \frac{\partial \Omega}{\partial p}\right)
\end{aligned}
$$

where $p=|\mathbf{p}|$ and

$$
\mathcal{H}=a^{-1} \frac{d}{d t} a=a H
$$


Here, $H$ is the Hubble expansion rate.

We have shown in [25] that if the phase space distribution has the BoseEinstein or Fermi-Dirac equilibrium limit which is a minimum of the relative entropy (related to the free energy ) then the diffusion equation must be nonlinear. The proper generalization of eq.(13) (which leads to the equilibrium in a static metric) reads

$$
\left(p^{\mu} \partial_{\mu}^{x}-\Gamma_{\mu \nu}^{k} p^{\mu} p^{\nu} \partial_{k}\right) \Omega=\kappa^{2} p^{0} \partial_{j}\left(g^{j k} \frac{1}{p^{0}} \partial_{k} \Omega+\beta a^{2} p^{j} \Omega(1+\nu \Omega)\right),
$$

where $\nu=1$ for bosons and $\nu=-1$ for fermions. The classical (Boltzmann) statistics can be described by $\nu=0$. The relation to Kompaneets equation [26][27]is discussed in [25].

In the ultrarelativistic limit $m=0$ the dependence of the diffusion generator (11) on the metric $h_{j k}$ disappears. In such a case the quantum equilibrium distributions solve the diffusion equation (19) in an expanding metric (8). In the case $m=0$ and for rotation invariant functions eq.(19) is

$$
\partial_{t} \Omega=2 \mathcal{H} p \frac{\partial \Omega}{\partial p}+\kappa^{2} p^{-2} \frac{\partial}{\partial p} p^{3}\left(\frac{\partial \Omega}{\partial p}+\beta a^{2} \Omega(1+\nu \Omega)\right) .
$$

In the ultrarelativistic limit at low density the dependence on the statistics should be irrelevant. We can neglect the quadratic term in eq. $(20)(\nu=0)$. Eq.(20) becomes linear

$$
\partial_{t} \Omega=\kappa^{2} p \frac{\partial^{2} \Omega}{\partial p^{2}}+\left(3 \kappa^{2}+2 \mathcal{H} p+\beta \kappa^{2} a^{2} p\right) \frac{\partial \Omega}{\partial p}+3 \beta \kappa^{2} a^{2} \Omega .
$$

In the initial stage of the expansion the frequent particles' interactions allow to achieve an equilibrium. The quantum equilibrium distribution will be a consequence of eq.(20). In the ultrarelativistic regime the classical approximation should apply leading to the Jüttner equilibrium distribution [20] . In fact, the $\beta$-dependent term in eq.(21) can be obtained from the principle of the detailed balance under the assumption that at infinite time $\Omega$ should reach the Jüttner equilibrium distribution.

The assumption $m=0$ can be justified in grand unified theories at high temperatures. The approximation $p^{0}=|\mathbf{p}|$ at a later time can be treated as a zeroth order term in an expansion of the solutions of eqs.(12)-(13) in powers of $\frac{m}{a p}$. Eq.(17) tends to the massless limit (21) when $m^{2} a^{-2} p^{-2} \rightarrow 0$. In the sequel we first restrict ourselves to the massless (high energy) limit of both eqs.(12)(13). In sec.7 a non-relativistic limit (large mass limit) of eq.(12) is applied for a description of the final stage of the Universe evolution as a cold dust.

Eq.(13) can be generalized to a non-isotropic Bianchi space-time with the metric

$$
d s^{2}=d \tau^{2}-a_{1}^{2}\left(d x^{1}\right)^{2}-a_{2}^{2}\left(d x^{2}\right)^{2}-a_{3}^{2}\left(d x^{3}\right)^{2} \equiv h_{j k} d x^{j} d x^{k}
$$


where $a_{j}$ depend only on $\tau$. The generalization (for massless particles) takes the form

$$
\left(p^{\mu} \partial_{\mu}^{x}-\Gamma_{\mu \nu}^{k} p^{\mu} p^{\nu} \partial_{k}\right) \Omega=\kappa^{2} p^{0} \partial_{j}\left(g^{j k} \frac{1}{p^{0}} \partial_{k}+\beta p^{j} \frac{1}{p^{0}} \sqrt{\sum_{l} p_{l}^{2} a_{l}^{4}}\right) \Omega .
$$

where in the cosmic time $p^{0}=\sqrt{h_{j k} p^{j} p^{k}}$.

\section{Solutions of linear and non-linear diffusion equa- tions at finite temperature}

In a time-independent metric any solution of the diffusion equations (13) or (19) tends to an equilibrium which is a time-independent solution of these equations. We are looking for analogs of equilibrium solutions in the case of an expanding flat Universe. It is easy to see that the time dependent Planck distribution $\Omega_{E}^{P L}$ is the solution of eq.(20). We have

$$
\Omega_{E}^{P L}=\left(\exp \left(\beta a^{2}(p+\mu)\right)-\nu\right)^{-1}
$$

where $\mu$ is an arbitrary constant (the chemical potential). In the ultrarelativistic limit ( a large $p$ ) the Planck distribution (23) is the same as the Jüttner distribution. However, the CMB measurements [31] show that the distribution (23) is valid also for a small $p$. We are unable to derive other solutions (starting from other initial phase space distributions) of the non-linear diffusion equation (20). Further on we discuss only the linear approximation (13) which can be considered as the ultrareletivistic limit of eq.(20).

We are looking for a general class of solutions of the linear diffusion equation (13). We show that besides the time dependent Jüttner distribution there are more general solutions which preserve their form under the time evolution. Hence, they could be considered as analogs of the equilibrium distribution. We show that the energy-momentum tensor (2) corresponding to such solutions depends on the scale factor $a$ in a more complicated way than it has been discussed in the literature so far ( see [44]). The main conclusion of this section is that the solution depends on an integral $A$ of $a^{2}$. In subsequent sections we investigate the dependence of the energy-momentum on $a$ and $A$. We show that the dependence on $A$ can be neglected at a large time.

First, we look for solutions of eq.(21) in an exponential form generalizing the Jüttner distribution. Then, we show that an initial distribution which is of the Jüttner form multiplied by a polynomial in $p$ preserves its shape under the time evolution. Such momentum distributions could be considered as analogs of the equilibrium distributions for a time-dependent metric. Assume the solution of eq.(21) has the form

$$
\Omega_{E}^{\beta}(t)=L(t) \exp (-\alpha(t) p) .
$$


Then,

$$
\partial_{t} L=-3 \kappa^{2} \alpha L+3 \beta \kappa^{2} a^{2} L
$$

and

$$
-\partial_{t} \alpha=\kappa^{2} \alpha^{2}-2 H \alpha-\beta \kappa^{2} a^{2} \alpha .
$$

The solution of eq.(25) with the initial condition $L\left(t_{0}\right)=1$ is

$$
L=\exp \left(-3 \kappa^{2} \int_{t_{0}}^{t} \alpha+3 \beta \kappa^{2} A\right)
$$

where

$$
A(t)=\int_{t_{0}}^{t} a^{2}(\tau(r)) d r
$$

Let

$$
w=\exp \left(\kappa^{2} \int \alpha\right)
$$

and $v=\partial_{t} w$. Then, from eq.(26)

$$
v=b a^{2}(t) \exp \left(\beta \kappa^{2} A\right)
$$

with a certain constant $b$. Hence, from eq.(29)

$$
\alpha=\beta a^{2} \exp \left(\kappa^{2} \beta A\right)\left(R+\exp \left(\kappa^{2} \beta A\right)\right)^{-1}
$$

where from the initial condition we can determine $R$

$$
\alpha\left(t_{0}\right)=\beta a^{2}\left(t_{0}\right)(R+1)^{-1} .
$$

If the initial condition $\alpha\left(t_{0}\right)=\beta a^{2}\left(t_{0}\right)$ is chosen then $R=0$ and the solution (24) for any $t$ is

$$
\Omega_{E}^{J}(t)=\exp \left(-\beta a^{2} p\right)
$$

(the Jüttner distribution [20]). Moreover, for any initial condition if $a(t) \rightarrow \infty$ when $t \rightarrow \infty$, then for $\Omega_{E}^{\beta}$ of eq.(24)

$$
\left|\Omega_{E}^{\beta}-\exp \left(-\beta a^{2}(t) p\right)\right| \rightarrow 0
$$

exponentially fast ( as from eq.(31) $\alpha \rightarrow \beta a^{2}$ and from eq.(27) $L \rightarrow 1$ ).

Let

$$
p=u^{2} .
$$

Then, eq.(21) takes the form

$$
\partial_{t} \Omega=\frac{\kappa^{2}}{4} \frac{\partial^{2} \Omega}{\partial u^{2}}+\frac{5 \kappa^{2}}{4} u^{-1} \frac{\partial \Omega}{\partial u}+\frac{1}{2}\left(2 \mathcal{H}+\beta \kappa^{2} a^{2}\right) u \frac{\partial \Omega}{\partial u}+3 \beta \kappa^{2} a^{2} \Omega .
$$


In order to obtain another set of solutions perturbing the solution (33) we write

$$
\Omega=\Omega_{E}^{J} \Psi .
$$

We can express eq.(36) for $\Psi$ as an equation in 6 dimensional momentum space $\mathbf{q}$ with $\mathbf{q}^{2}=u^{2}=p$ as follows

$$
\partial_{t} \Psi=\frac{\kappa^{2}}{4} \triangle_{6} \Psi+\frac{1}{2} \omega_{\beta} \mathbf{q} \nabla \Psi
$$

where $\triangle_{6}$ is the Laplacian in a 6 dimensional momentum space $R^{6}$ and

$$
\omega_{\beta}=2 \mathcal{H}-\beta \kappa^{2} a^{2}
$$

Rotation invariant solutions of eq.(38) solve eqs.(36)-(37). In the space of rotation invariant functions we can introduce a polynomial basis

$$
\Psi(t, \mathbf{q})=1+\sum_{n=0}^{n=N} c_{n}(t)\left(\mathbf{q}^{2}\right)^{n}
$$

Inserting eq.(40) in eq.(38) we find linear equations for $c_{n}$

$$
\begin{gathered}
\partial_{t} c_{N}=N \omega_{\beta} c_{N}, \\
\partial_{t} c_{n-1}=(n-1) \omega_{\beta} c_{n-1}+\kappa^{2} n(n+2) c_{n}
\end{gathered}
$$

with $c_{0}\left(t_{0}\right)=0$. These equations can be solved systematically starting from $N$. First

$$
c_{N}\left(t, t_{0}\right)=c_{N}\left(t_{0}\right) a(t)^{2 N} a\left(t_{0}\right)^{-2 N} \exp \left(-N \kappa^{2} \beta A(t)\right) .
$$

Then, we can find the coefficients for lower $n$. In detail, for $N=1$

$$
c_{0}(t)=\frac{3}{\beta} a\left(t_{0}\right)^{-2}\left(1-\exp \left(-\kappa^{2} \beta A(t)\right)\right) c_{1}\left(t_{0}\right)
$$

and

$$
c_{1}\left(t, t_{0}\right)=c_{1}\left(t_{0}\right) a^{2}(t) a\left(t_{0}\right)^{-2} \exp \left(-\kappa^{2} \beta A(t)\right) \equiv \tilde{c}_{1} a^{2}(t) .
$$

For $N=2$ we have

$$
\begin{gathered}
c_{2}\left(t, t_{0}\right)=c_{2}\left(t_{0}\right) \frac{a(t)^{4}}{a\left(t_{0}\right)^{4}} \exp \left(-2 \kappa^{2} \beta A\right) \equiv \tilde{c}_{2} a^{4}, \\
c_{1}\left(t, t_{0}\right)=\frac{a^{2}(t)}{a^{2}\left(t_{0}\right)} \exp \left(-\beta \kappa^{2} A\right) c_{1}\left(t_{0}\right) \\
+\frac{8}{\beta} \frac{a^{2}(t)}{a^{4}\left(t_{0}\right)} \exp \left(-\beta \kappa^{2} A\right)\left(1-\exp \left(-\beta \kappa^{2} A\right)\right) c_{2}\left(t_{0}\right) \equiv \tilde{c}_{1} a^{2}, \\
c_{0}\left(t, t_{0}\right)=\frac{12}{\beta^{2} a^{4}\left(t_{0}\right)}\left(1-\exp \left(-\beta \kappa^{2} A\right)\right)^{2} c_{2}\left(t_{0}\right) .
\end{gathered}
$$


From eqs.(41)-(42) we can derive the behaviour of $c_{n-1}$ as $\simeq a^{2(n-1)}$. In fact, we have

$$
c_{n-1}(t)=\exp \left(\int_{t_{0}}^{t}(n-1) \omega_{\beta}\right) c_{n-1}\left(t_{0}\right)+\kappa^{2} n(n+2) \int_{t_{0}}^{t} \exp \left(\int_{r}^{t}(n-1) \omega_{\beta}\right) c_{n}(r) d r .
$$

Now, if $c_{n} \simeq a^{2 n}$ then $\exp \left((n-1) \int_{t_{0}}^{t} \omega_{\beta}\right) \simeq a(t)^{2(n-1)} a\left(t_{0}\right)^{-2(n-1)}$ and $\exp ((n-$ 1) $\left.\int_{r}^{t} \omega_{\beta}\right) c_{n}(r) \simeq a(t)^{2(n-1)} a^{2}(r)$. It follows that $c_{n-1}(t) \simeq a^{2(n-1)}(t) f(A(t))$ with a certain bounded function $f$. Hence, from the $c_{n} \simeq a^{2 n}$ behaviour we obtain the behaviour $c_{n-1} \simeq a^{2(n-1)}$ and inductively for any lower $n$. It can also be seen from eqs.(41)-(42) that we have $c_{n} \rightarrow 0$ (for $n>0$ ) because these coefficients $(\simeq f(A))$ decay exponentially with a growing $A$ and a multiplication by powers of $a$ does not change the limit $t \rightarrow \infty$.

The diffusion equation (22) for the Bianchi space-time has a $\kappa$-independent solution $\Omega_{E}^{B}$ which is an analog of the Jüttner solution (33)

$$
\Omega_{E}^{B}=\exp \left(-\beta \sqrt{a_{1}^{4} p_{1}^{2}+a_{2}^{4} p_{2}^{2}+a_{3}^{4} p_{3}^{2}}\right) .
$$

However, in the anisotropic case we are unable to find analogs of the $\kappa$-dependent solutions which we have discussed in this section and the ones to be considered in the next section. Nevertheless, the distribution $\Omega_{E}^{B}$ is interesting as an example leading to an anisotropic energy-momentum.

\section{A transition from finite temperature to zero temperature}

There are complex quantum processes involved in the early Universe. We have at our disposal classical Einstein equations. We assume that the momentum distribution of particles in the Universe soon after the Big Bang can be described by the non-linear diffusion equation for massless particles. We suggest that (like in the linear case (34)) the solution of the non-linear equation starting from an arbitrary initial condition is approaching for a later time the time-dependent Planck distribution. In an early stage of the Universe evolution eq.(19) describes the diffusive behaviour of the phase space distribution leading to the Planck distribution (23). In the ultrarelativistic approximation we restrict ourselves to the linear equation (19) with the Jüttner equilibrium distribution. After some time $t_{0}$ (beginning of the lepton era) new forms of matter are created weakly interacting with radiation. We do not expect that Jüttner phase space distribution remains valid for all kinds of matter at a later time. We shall describe the subsequent time evolution beginning at $t=t_{0}$ at the expansion scale $a\left(t_{0}\right)$ and at the temperature $\beta^{-1}=\kappa^{2} \theta\left(a^{2}\left(t_{0}\right)\right)^{-1}$ by the diffusion equation (12) corresponding to a diffusion at zero temperature with no equilibration. The solutions of eq.(21) on the time interval $\left[0, t_{0}\right)$ can be transferred continuously 
to solutions of eq.(12) on the interval $\left[t_{0}, \infty\right)$. The diffusion constant $\kappa$ in eq. (21) is determined by scattering processes of the hot matter in the early Universe. It is not the same as the diffusion constant in eq.(12) relevant for a later time when scattering processes of different particles formed at later time of the evolution are involved.

In this section we follow the procedure of the previous section finding first a solution of the diffusion equation (12) of an exponential (Jüttner) type. Subsequently, we obtain analogs of the solutions (37) obtained by a polynomial perturbation of the exponential solution. The parameter $\beta$ in eq.(21) is to disappear at $t=t_{0}$ when the phase space distribution begins to evolve according to eq.(12). If $\beta \rightarrow 0$ in eq.(31) then (with $1+R=\theta \beta \kappa^{2}$ ) we obtain

$$
\alpha=\kappa^{-2} a^{2}(\theta+A)^{-1} .
$$

In this way we find a particular solution of eq. $(21)(\beta=0)$ starting from the Jüttner distribution $\Omega_{0}$ at $t=t_{0}$

$$
\Omega_{0}=\exp \left(-\left(\kappa^{2} \theta\right)^{-1} a^{2}\left(t_{0}\right) p\right)
$$

(the parameter $\kappa^{2} \theta\left(a^{2}\left(t_{0}\right)\right)^{-1}$ has the meaning of temperature) then continuing at $t \geq t_{0}$ as

$$
\Omega_{E}(t)=\theta^{3}(\theta+A)^{-3} \exp \left(-\kappa^{-2} \frac{a^{2}}{\theta+A} p\right) .
$$

$\Omega_{E}(t)$ solves eq.(12) (eq.(21) with $\beta=0$ ) with the initial condition $\Omega_{0}$.

If the diffusion (12) starts from the Planck distribution $(23)(\mu=0)$ then it continues as

$$
\Omega_{E}^{P}(t)=\sum_{k=1}^{\infty} \nu^{k-1}\left(1+\kappa^{2} k \beta A\right)^{-3} \exp \left(-\frac{a^{2} \beta k}{1+\kappa^{2} k \beta A} p\right)
$$

At large momenta the terms with $k>1$ in eq.(50) are negligible. We treat the Jüttner-type solution (49) at zero temperature as the high-energy approximation to the Planck-type solution (50).

In the Jüttner case we can insert again eq.(37) into eq.(36) with $\beta=0$ and derive eqs.(41)-(42) with

$$
\omega=-\frac{d}{d t} \ln \left(\frac{\theta+A}{a^{2}}\right)
$$

We have solved eqs.(41)-(42) explicitly for small $N$. We have

$$
c_{N}\left(t, t_{0}\right)=c_{N}\left(t_{0}\right) a(t)^{2 N} a\left(t_{0}\right)^{-2 N}(\theta+A)^{-N} \theta^{N} .
$$

Then, for $N=1$

$$
c_{1}(t)=\frac{a^{2}(t)}{a^{2}\left(t_{0}\right)}(\theta+A)^{-1} \theta c_{1}\left(t_{0}\right) \equiv \tilde{c}_{1} a^{2}(t)(\theta+A)^{-1},
$$




$$
c_{0}(t)=3 c_{1}\left(t_{0}\right) \kappa^{2} \theta \ln \left(1+\frac{A}{\theta}\right) .
$$

For $N=2$ we have

$$
\begin{gathered}
c_{2}(t)=\frac{a(t)^{4}}{a^{4}\left(t_{0}\right)}(\theta+A)^{-2} \theta^{2} c_{2}\left(t_{0}\right) \equiv \tilde{c}_{2} a^{4}(t)(\theta+A)^{-2} \\
c_{1}(t)=\frac{a^{2}(t)}{a^{2}\left(t_{0}\right)}(\theta+A)^{-1} \theta c_{1}\left(t_{0}\right)+8 c_{2}\left(t_{0}\right) \kappa^{2} a^{2}(t) a\left(t_{0}\right)^{-4} \theta^{2}(\theta+A)^{-1} \ln \left(1+\frac{A}{\theta}\right) \\
\equiv \tilde{c}_{1} a^{2}(t)(\theta+A)^{-1} \\
c_{0}(t)=3 c_{1}\left(t_{0}\right) \kappa^{2} a\left(t_{0}\right)^{-2} \theta \ln \left(1+\frac{A}{\theta}\right)+24 \kappa^{4} \theta^{2} c_{2}\left(t_{0}\right) a\left(t_{0}\right)^{-4}\left(\ln \left(1+\frac{A}{\theta}\right)\right)^{2}
\end{gathered}
$$

The solutions (52)-(57) will be discussed in subsequent sections.

\section{Einstein equations for diffusing particles at high temperature}

In the remaining sections we discuss the Einstein equations (1). In principle, we should discuss all fields and particles entering the energy-momentum and consider a quantum version of Einstein equations. However, to be realistic we accept the point of view that we are far from a knowledge of all forms of matter and energy and their interaction. Having a partial knowledge we approximate the impact of the unknown interactions by a diffusion whereas the energy-momentum of unknown particles and interactions is described by $T_{D}^{\mu \nu}$ in eq.(5). The conservation of the total energy-momentum determines $T_{D}$ in terms of the energy-momentum $\tilde{T}$ of diffusing particles according to eq.(6). An indication of a necessity of such an approach is the coincidence problem [37](the dark matter and luminous matter are of the same order). The main aim of this section is a derivation of $T_{D}$ from eq.(6). For a homogeneous and isotropic Universe this will allow us to write down the Einstein equations (5) for the scale factor $a$ (the Friedmann equations) explicitly. We shall derive the dependence of the energy-momentum on the scale factor $a$ using the solutions of the diffusion equation obtained in secs. 3 and 4 . We discuss the behaviour of solutions of the Friedmann equations from the point of view of the energy conditions satisfied by $\tilde{T}+T_{D}$.

We calculate the energy-momentum using the phase space distribution resulting from the solution of the diffusion equation. In the early Universe this should be the non-linear diffusion leading to the experimentally confirmed [31] CMB distribution (23). In eq.(4) we have already inserted the division of the quantum phase space into elementary units of the volume $(2 \pi \hbar)^{3}$ ( we set $\hbar=1$ ). The solutions (23) and (33) are expressed in the comoving frame (moving with the fluid). We restrict ourselves in this section to the linear approximation of 
the diffusion equation (20) at finite temperature ( without the heat bath we have always the linear equation (12)). If in a new frame the fluid is moving with a velocity $w\left(w^{\mu} w_{\mu}=1\right)$ then in this frame the time is $s=w_{\mu} x^{\mu}$ and the energy $p^{\mu} w_{\mu}$. The scale factor $a$ depends on $s . \Omega_{E}^{J}(33)$ is expressed in a covariant form as

$$
\Omega_{E, n}^{J}=n M^{-1} \exp \left(-\beta a(s) w_{\mu} p^{\mu}\right),
$$

where $n$ and $M$ are normalization factors. The normalization is introduced [45] according to the rule that the normalized state $\Omega_{n}$ satisfies the condition

$$
\sqrt{h} \int \frac{d \mathbf{p}}{(2 \pi)^{3}} \Omega_{n}=n .
$$

Here, $n$ is the density defined by the current [13][41]

$$
N^{\mu} \equiv n u^{\mu}=\sqrt{h} \int \frac{d \mathbf{p}}{(2 \pi)^{3}} \frac{1}{p_{0}} p^{\mu} \Omega_{n} .
$$

which determines the density $n$ and the velocity $u$ of the fluid ( $u^{\mu} u_{\mu}=1$ ). If for an unnormalized state $\Omega$ we define

$$
M=\sqrt{h} \int \frac{d \mathbf{p}}{(2 \pi)^{3}} \Omega,
$$

then the normalization of an arbitrary distribution $\Omega$ is

$$
\Omega_{n}=n M^{-1} \Omega \text {. }
$$

We define the energy-momentum tensor as [13] [41]

$$
\tilde{T}^{\mu \nu}=\sqrt{h} \int \frac{d \mathbf{p}}{(2 \pi)^{3}} \frac{1}{p_{0}} p^{\mu} p^{\nu} \Omega_{n} .
$$

We are going to study the Universe evolution described by the states (37) either for a large or for a small time. It is usually discussed in the cosmic time $\tau$. Note that in conformal time $\sqrt{h}=a^{4}, p_{0}=|\mathbf{p}|$, whereas in cosmic time $\sqrt{h}=a^{3}$ and $p_{0}=a|\mathbf{p}|$. From now on we express all equations in the cosmic time. If there is no diffusion then $\Omega$ satisfies the Liouville equation (3). The energy-momentum tensor is conserved. This is the case for $\Omega_{E}$ of eqs.(23) and (33). In this state both sides of eq.(13) are equal to zero. Hence, eq.(3) is satisfied. The formula (63) gives $\tilde{T}^{00} \simeq a^{-4}$ in the states (23) and (33). In the Jüttner state (23) we obtain from eq.(1) the Friedmann equation (flat space, cosmic time)

$$
\left(a^{-1} \frac{d a}{d \tau}\right)^{2}=\frac{8 \pi G}{3} \frac{1}{(2 \pi)^{3}} 24 \pi(\beta a)^{-4} .
$$

In the Planck state (33) there will be an extra factor $\frac{\pi^{4}}{90} \simeq 1$ on the rhs of eq.(64) corresponding to the correction for small momenta contribution to the integral 
(63). The resulting Friedmann equation coincides with the standard one (see [41], sec.1.3).

The energy-momentum tensor (63) is not conserved if the solution of the diffusion equation depends on $\kappa$ as the general solutions of secs. 3 and 4 do. In general, for massless particles we have [17][32]

$$
\left(\tilde{T}^{\mu \nu}\right)_{; \mu}=3 \kappa^{2} N^{\nu}-\beta \kappa^{2} \tilde{T}^{0 \nu} .
$$

Using the Christoffel symbols (15)-(16) the covariant divergence of an arbitrary tensor $T$ reads

$$
\left(T^{\mu 0}\right)_{; \mu}=\partial_{\tau} T^{00}+3 a \frac{d a}{d \tau} T^{00}+a \frac{d a}{d \tau} \delta_{j k} T^{j k} .
$$

In a homogeneous Universe

$$
\tilde{T}^{\mu \nu}=\tilde{E} u^{\mu} u^{\nu}-\tilde{\pi}_{E}\left(h^{\mu \nu}-u^{\mu} u^{\nu}\right),
$$

where $\tilde{E}$ is the energy, $\tilde{\pi}_{E}$ the pressure and the four-velocity $u^{\mu}$ satisfies the condition

$$
h_{\mu \nu} u^{\mu} u^{\nu}=1 .
$$

For massless particles $\tilde{T}_{\mu}^{\mu}=0$. Hence,

$$
\tilde{\pi}_{E}=\frac{1}{3} \tilde{E}
$$

In the frame $u=(1,0)$ we have

$$
\left(\tilde{T}^{\mu 0}\right)_{; \mu}=\partial_{\tau} \tilde{E}+3 a^{-1} \partial_{\tau} a\left(\tilde{E}+\tilde{\pi}_{E}\right) .
$$

We shall represent the unknown energy $T_{D}$ in eq.(5) by a cosmological term $\Lambda$. Then

$$
T^{\mu \nu}=\tilde{T}^{\mu \nu}+h^{\mu \nu} \frac{\Lambda}{8 \pi G},
$$

where $\tilde{T}$ is the energy-momentum tensor (63). The energy conservation (2) is expressed as

$$
-\partial_{\tau} \frac{\Lambda}{8 \pi G}=\partial_{\tau} \tilde{E}+3 a^{-1} \partial_{\tau} a\left(\tilde{E}+\tilde{\pi}_{E}\right)
$$

In the massless case (68) eq.(71) gives

$$
\frac{\Lambda(\tau)}{8 \pi G}=\frac{\Lambda\left(\tau_{0}\right)}{8 \pi G}-a^{-4} \int_{\tau_{0}}^{\tau} \partial_{r}\left(a^{4} \tilde{T}^{00}\right) d r
$$

$\Lambda$ is determined from eq.(71) up to a constant. We could also integrate eq.(65) in order to express $\Lambda$ by $\tilde{T}$ and $N^{\mu}$. From eq.(65) it can be seen that the 
divergence of $\tilde{T}^{\mu \nu}$ is proportional to the diffusion constant. Applying eq.(72) we obtain the Einstein equations (for a flat Universe) in the form

$$
\begin{aligned}
& \frac{3}{8 \pi G} H^{2} \equiv \frac{3}{8 \pi G}\left(a^{-1} \frac{d a}{d \tau}\right)^{2}=\tilde{T}^{00}(\tau)-\int_{\tau_{0}}^{\tau} d r a^{-4} \partial_{r}\left(a^{4} \tilde{T}^{00}\right)+\frac{\Lambda}{8 \pi G}\left(\tau_{0}\right) \\
& =\tilde{T}^{00}\left(\tau_{0}\right)-4 \int_{\tau_{0}}^{\tau} d r H(r) \tilde{T}^{00}(r)+\frac{\Lambda}{8 \pi G}\left(\tau_{0}\right)
\end{aligned}
$$

It follows from eq.(73) that the modification of the Friedmann equation resulting from the diffusion is determined by the deviation of $\tilde{T}^{00}$ from the standard $a^{-4}$ (as in eq.(64)).

Eq.(73) can also be expressed in the form

$$
\frac{3}{8 \pi G}\left(H^{2}(\tau)-H^{2}\left(\tau_{0}\right)\right)=-4 \int_{\tau_{0}}^{\tau} d r H(r) \tilde{T}^{00}(r),
$$

where we have used the relation

$$
\frac{3}{8 \pi G} H^{2}\left(\tau_{0}\right)=\tilde{T}^{00}\left(\tau_{0}\right)+\frac{\Lambda}{8 \pi G}\left(\tau_{0}\right)=\tilde{E}\left(\tau_{0}\right)+\frac{\Lambda}{8 \pi G}\left(\tau_{0}\right)
$$

resulting from the notation of eqs.(66) and (70)

$$
T^{00}=\tilde{E}+\frac{\Lambda}{8 \pi G} .
$$

If we know $H$ at a certain moment $\tau_{0}$ then eq. (74) determines $a(\tau)$ for a later time. Note that if $\frac{d a}{d \tau} \geq 0$ for $\tau \geq \tau_{0}$ then from eq.(73) it follows that with

$$
\frac{3}{8 \pi G} \lambda \equiv \tilde{E}\left(\tau_{0}\right)+\frac{\Lambda}{8 \pi G}\left(\tau_{0}\right)-4 \int_{\tau_{0}}^{\infty} d r H(r) \tilde{T}^{00}(r)
$$

we have the bounds

$$
\lambda \leq\left(a^{-1} \frac{d a}{d \tau}\right)^{2} \leq \frac{8 \pi G}{3} \tilde{E}\left(\tau_{0}\right)+\frac{\Lambda}{3}\left(\tau_{0}\right)
$$

If $\lambda$ of eq.(76) is non-negative then we obtain the inequality (Gronwall inequality)

$$
\exp \left(\tau H\left(\tau_{0}\right)\right) \geq a(\tau) \geq \exp (\sqrt{\lambda} \tau)-\exp \left(\sqrt{\lambda} \tau_{0}\right) .
$$

There can be an exponential behaviour in a certain time interval when the cosmological constant dominates and a power-like behaviour if the cosmological constant is cancelled by other terms on the rhs of eq.(73).

It is useful to study the behaviour of solutions of Einstein equations from the point of view of the energy conditions [38] [46]. We can represent $T^{\mu \nu}$ again in the hydrodynamic form (66) (with $E$ and $\Pi_{E}$ without tilde). We have already assumed that we have as a solution a flat Universe. This is possible only if $E=T^{00}=\tilde{T}^{00}+\frac{\Lambda}{8 \pi G} \geq 0$. In the hydrodynamic representation $\tilde{\Pi}=\frac{1}{3} \tilde{E}$ because $p^{2}=0$ (eq.(68)). We can conclude from eqs.(66) and (70) that

$$
E+3 \Pi_{E}=2\left(\tilde{E}-\frac{\Lambda}{8 \pi G}\right)
$$


We have $E+\Pi_{E}=\tilde{E}+\tilde{\Pi}_{E}, \Pi_{E}=\tilde{\Pi}_{E}-\frac{\Lambda}{8 \pi G}$ and $E=\tilde{E}+\frac{\Lambda}{8 \pi G}$. From eq.(78) we can see that the strong energy condition (requiring that (78) be positive [38])can be violated if the cosmological term is present. Using the definition of $\lambda$ (eq.(76)) we can obtain (under the assumption $\partial_{\tau} a \geq 0$ for $\tau \geq \tau_{0}$ )

$$
\tilde{E}(\tau)-\frac{\Lambda(\tau)}{8 \pi G} \leq 2 \tilde{E}(\tau)-\frac{3 \lambda}{8 \pi G}
$$

In cosmological models the energy density $\tilde{E}(\tau)$ is decreasing to zero when the Universe expands. Hence, if $\lambda$ is positive then according to eqs.(78)-(79) the strong energy condition will be violated for a sufficiently large time. From Einstein equations $\partial_{\tau}^{2} a \simeq-a\left(E+3 \Pi_{E}\right)$. Hence, (78) will be negative if the expansion accelerates. This explains the role played by the positivity of $\lambda$ in eqs.(77) and (78).

It is clear that the weak energy condition $E \geq 0$ and $E+\Pi_{E} \geq 0$ is satisfied. The dominant energy condition is equivalent to $E=\tilde{E}+\frac{\Lambda}{8 \pi G} \geq\left|\Pi_{E}\right|=\mid \frac{1}{3} \tilde{E}-$ $\frac{\Lambda}{8 \pi G} \mid$. Using eq.(72) this inequality can be rewritten as either $\Lambda \geq-\frac{8 \pi G}{3} \tilde{E}(\tau)$ or equivalently as

$$
\tilde{E}\left(\tau_{0}\right)+\frac{\Lambda\left(\tau_{0}\right)}{8 \pi G} \geq \frac{2}{3} \tilde{E}(\tau)+4 \int_{\tau_{0}}^{\tau} d r H(r) \tilde{T}^{00}(r) .
$$

In cosmological models $\tilde{E}(\tau)$ is decreasing to zero for a large time. Hence, we can obtain a sufficient condition for the dominant energy condition to be satisfied for a sufficiently large $\tau$

$$
\tilde{E}\left(\tau_{0}\right)+\frac{\Lambda\left(\tau_{0}\right)}{8 \pi G}-4 \int_{\tau_{0}}^{\infty} d r H(r) \tilde{T}^{00}(r)=\frac{3}{8 \pi G} \lambda>0 .
$$

If $\lambda>0$ then the strong energy condition is violated whereas the dominant energy condition is satisfied. It can be seen that both conditions depend on the initial value for the diffusion phase space distribution and the initial value of the cosmological term.

Einstein equations with the energy-momentum calculated in the states at finite temperature are applied only at small time. If $a(\tau)$ tends to zero at small $\tau$ then we can derive the behaviour of $a(\tau)$ from the behaviour of the energymomentum for a small $a$. We calculate the energy-momentum in the states of sec.3. For the state (31) we obtain $\left(\right.$ here $\sqrt{h}=h^{3}$ )

$\tilde{T}^{00}=L(t) \sqrt{h} \int \frac{d \mathbf{p}}{(2 \pi)^{3}} a p \exp (-\alpha(t) p)=L(t) \frac{1}{(2 \pi)^{3}} 24 \pi(\beta a)^{-4}\left(1+R \exp \left(-\kappa^{2} \beta A\right)\right)^{4}$

For the state (44)-(45) we have

$$
\tilde{T}^{00}=\frac{1}{(2 \pi)^{3}}\left(24 \pi(\beta a)^{-4}\left(1+c_{0}(1)\right)+96 \pi(\beta a)^{-5} \tilde{c}_{1}(1)\right)
$$


and for (46)-(47)

$$
\begin{aligned}
& \tilde{T}^{00}=\frac{1}{(2 \pi)^{3}}\left(24 \pi(\beta a)^{-4}\left(1+c_{0}(2)\right)+96 \pi(\beta a)^{-5} \tilde{c}_{1}(2)\right. \\
& \left.+480 \tilde{c}_{2}(2)(\beta a)^{-6}\right)
\end{aligned}
$$

We can calculate the remaining components of $\tilde{T}^{\mu \nu}$ in the comoving frame $u=$ $(1,0)$. We have according to eq. (66) $\tilde{E}=\tilde{T}^{00}, \tilde{T}^{0 j}=0$ and owing to the rotational symmetry $\tilde{T}^{j k}=\frac{1}{3} \delta^{j k} \tilde{T}^{00}$. Then, from eq.(68) $\tilde{\Pi}_{E}=\frac{1}{3} \tilde{T}^{00}$ in the hydrodynamic representation (66) .

The coefficients in eqs.(82)-(83) (where $c(2)$ means that this is $c$ at $N=2$ ) depend on $A$ in such a way that if $A$ tends to infinity at large time then the coefficients $\tilde{c}_{1}$ and $\tilde{c}_{2}$ tend to zero. Hence, we do not expect a modification of the large time behaviour at non-zero temperature in the states (37). The argument at the end of sec.3 shows that the results (81)-(83) can be extended to general $N$

$$
\tilde{T}^{00}=(\beta a)^{-4} \sum_{n=0}^{n=N} \tilde{c}_{n}(\beta a)^{-n}
$$

with $\tilde{c}_{n}$ (for $n>0$ ) decaying as $\exp \left(-\beta \kappa^{2} A\right.$ ). For general $N$ we obtain again the conclusion that the polynomial modification (37) of the Jüttner distribution does not change the large time behaviour of the energy-momentum in the Einstein equations (note however that according to sec.4 at sufficiently large time the temperature states are replaced by (49), their energy-momentum is discussed in the next section). The dissipative modification (73) of the Friedmann equations comes from a departure of $a^{4} \tilde{T}^{00}$ from a constant (the constant is obtained for a radiation without dissipation). From eq.(72) it follows that $\Lambda$ is decreasing if $a^{4} \tilde{T}^{00}$ is increasing (as will happen in the model (85) in the next section). The time dependent terms $\tilde{c}_{n}$ disappear at large time but they can be relevant for a rapid increase of $a(\tau)$ at an intermediate time. The terms $\tilde{c}_{n} a^{-n}$ in eq.(84) modify the short time behaviour of solutions of the Friedmann equations as will be shown at the end of the next section.

\section{Universe expansion at zero temperature}

In our approach the energy-momentum tensor is defined by the solution of the diffusion equation. We use different diffusion equations at various stages of the expansion of the Universe. In addition, the solution of the diffusion equation depends on the initial condition. The solutions of these equations determine different energy-momenta. In a static metric a solution of the diffusion equation tends to the unique equilibrium. The energy-momentum tends to the one calculated in the equilibrium. In a time-dependent metric strictly speaking an equilibrium solution does not exist. However, we can distinguish some initial phase space distributions which preserve their form during the time evolution. 
We could also require that at fixed time such states coincide with the static equilibrium states resulting from variational principles of statistical mechanics (as is the case with the Planck distribution $\Omega_{E}^{P L}(23)$ and the Jüttner distribution $\Omega_{E}^{J}(33)$ ). The distributions $\Omega_{E}^{P L}$ and $\Omega_{E}^{J}$ lead to the standard dependence of the energy-momentum $\tilde{T} \simeq a^{-4}$ on $a$ (in deterministic models it also follows from the continuity equation and the equation of state $E=w \Pi_{E}$, in the model (70) $w$ is not a constant; for a discussion of $a$-dependence of $\tilde{T}$ see [44]). We have seen in sec.5 that the modified Jüttner distribution leads to the modified energy momentum depending on $a$ as well as on $A$. However, the dependence on $A$ was irrelevant at large time. The solutions of the diffusion equation at finite temperature apply to an early stage of the evolution of the Universe. At later time $\tau \geq \tau_{0}$ (after the recombination time) the diffusion tending to an equilibrium cannot correctly describe the phase space distribution of particles which decouple from photons. In sec. 4 we have described a continuation of the evolution starting from the Jüttner (33)(or Planck (23)) state and continuing according to the dissipative dynamics (12) of the diffusion at zero temperature. In this section we calculate the energy-momentum $\tilde{T}$ of the solution of the diffusion equation (12). Subsequently, from the energy-momentum conservation we determine the cosmological term $T_{D}$. We derive the Friedmann equation and find a particular solution of this equation which is linear in time. At the end of this section we return to the finite temperature solutions of sec.5 in order to determine their behaviour at $a=0$.

In the zero temperature state (49) we obtain

$$
\tilde{T}^{00}=\sqrt{h} \theta^{3}(\theta+A)^{-3} \int \frac{d \mathbf{p}}{2 \pi)^{3}} a p \exp \left(-\kappa^{-2} \frac{a^{2}}{\theta+A} p\right)=\frac{1}{(2 \pi)^{3}} 24 \pi \kappa^{8} \theta^{3}(\theta+A) a^{-4} .
$$

For the state (53)-(54)

$$
\tilde{T}^{00}=\frac{1}{(2 \pi)^{3}} \kappa^{2}(\theta+A) a^{-4}\left(24 \pi\left(1+c_{0}(1)\right)+96 \pi a^{-1} \tilde{c}_{1}(1)\right)
$$

and for (55)-(57)

$$
\begin{aligned}
& \tilde{T}^{00}=\frac{1}{(2 \pi)^{3}} \kappa^{2} \theta^{-1}(\theta+A) a^{-4}\left(24 \pi\left(1+c_{0}(2)\right)+96 \pi a^{-1} \tilde{c}_{1}(2)\right. \\
& \left.+480 \pi \tilde{c}_{2}(2) a^{-2}\right)
\end{aligned}
$$

The model (85) leads (according to eq.(73)) to the Friedman equation

$$
\left(a^{-1} \frac{d a}{d \tau}\right)^{2}=\sigma(A+\theta) a^{-4}-\sigma \int_{\tau_{0}}^{\tau} d r a^{-3}+\frac{\Lambda}{3}\left(\tau_{0}\right)
$$

where

$$
\sigma=\frac{1}{(2 \pi)^{3}} 48 G \pi^{2} \kappa^{8} \theta^{3}
$$

We can find an explicit power-like solution of the integro-differential equation (88) by a fine tuning of parameters showing that the exponential behaviour (77) 
is not a necessity even if $\Lambda\left(\tau_{0}\right)>0$. Let us assume

$$
a(\tau)=\nu(\tau-q)^{\gamma}
$$

with the initial condition $a\left(\tau_{0}\right)=\nu\left(\tau_{0}-q\right)^{\gamma}$. Inserting (90) into eq.(88) we determine the parameters in eq.(90)

$$
\begin{gathered}
\gamma=1, \\
\nu=\sigma^{\frac{1}{3}}, \\
\left(\tau_{0}-q\right)^{2}=\frac{2 \theta}{\nu}
\end{gathered}
$$

and

$$
\Lambda\left(\tau_{0}\right)=\frac{3}{2}\left(\tau_{0}-q\right)^{-2}
$$

We can calculate the rhs of the Friedman equation which is $\frac{1}{3}(8 \pi G \tilde{E}+\Lambda)$. We obtain

$$
\Lambda=8 \pi G \tilde{E}=\frac{3}{2}(\tau-q)^{-2}
$$

Eq.(95) means that $E-3 \Pi_{E}=0$ in agreement with the discussion at eq.(78) (there is no deceleration).

Eq.(90) applies if $q<\tau_{0}$ because the integral in eq.(88) is divergent at $r=q$. The solution (90) defined on the interval $\left[\tau_{0}, \infty\right)$ does not achieve 0 reaching only its minimum at $a\left(\tau_{0}\right)=\nu\left(\tau_{0}-q\right)$. The solution (90) with $\gamma=1$ is interesting because it gives $H^{-1}$ (where $H$ is the present value of the Hubble constant) as the age of the Universe in agreement with recent experimental data ([47],sec.11.4.1; see also [48] [49] for an explanation of a distinguished character of the linear evolution).

For general $\tilde{T}^{00}$ resulting from eq.(40) we are unable to solve eq.(73) exactly. We can obtain some estimates on the asymptotic behaviour. We can have an exponential or power-like evolution depending on the parameters entering eq. (73). For a discussion of asymptotic solutions it is useful to differentiate the integro-differential equation (73). We obtain

$$
\frac{d}{d \tau}\left(a^{-1} \frac{d a}{d \tau}\right)=-\frac{16 \pi G}{3} \tilde{T}^{00}
$$

Note that differentiation of the standard Friedmann equation (64) (with $\tilde{T}^{00} \simeq$ $a^{-4}$ ) also gives eq.(96) but this coincidence is accidental. It results from the power -4 in $a^{-4}$. Nevertheless, if $\tilde{T}^{00} \simeq a^{-r}$ with $r>0$ then there is no essential difference between the standard Friedmann equation and (96) up to an undetermined cosmological constant which is anihilated when we differentiate the standard Friedmann equation. Eq.(96) can be rewritten as the third order equation for $A$ (with $A^{\prime}=\frac{d A}{d \tau}=a$ )

$$
A^{\prime \prime \prime}-\left(A^{\prime \prime}\right)^{2}\left(A^{\prime}\right)^{-1}=-\frac{16 \pi G}{3} T^{00}\left(A, A^{\prime}\right) A^{\prime} .
$$


It can be seen that the $a^{-n}$ terms in eq.(84) do not modify the large time behaviour because they decay faster in time. The Ansatz $A \simeq \tau^{\gamma+1}$ in eqs.(82)(84)and (97) for a large time gives again $\gamma$ of eq.(91).

For the early Universe we apply the finite temperature solutions of the diffusion equation of sec.5. We are interested in the small time behaviour of the solutions of the finite temperature diffusion on the time interval $\left[0, \tau_{0}\right)$. Using eq.(97) we can show that the polynomial modification (40) of the Jüttner initial condition changes the small time behaviour of the solutions of the Friedmann equations. At $a \simeq 0$ the most singular terms in $a$ dominate in eq.(84). Inserting the energy-momentum tensor (84) in the Friedmann equations (97) we obtain

$$
A^{\prime \prime \prime}-\left(A^{\prime \prime}\right)^{2}\left(A^{\prime}\right)^{-1} \simeq-C\left(A^{\prime}\right)^{-N-3}
$$

with a certain constant $C$. Eq.(98) leads to the behaviour

$$
a \simeq \tau^{\frac{2}{4+N}}
$$

when $\tau \rightarrow 0$. Hence, with an increasing $N$ the approach to the singularity $a=0$ becomes slower than in the exact solution (90) for $\tau>q$ and also slower than the $\sqrt{\tau}$ behaviour of the solution of the standard Friedmann equation (64). If the dependence of $T$ on $a$ is more singular than power-like then we can encounter solutions of the Friedmann equation (73) which do not reach $a=0$.

The solutions of secs. 3 and 4 could also describe an ultrarelativistic expanding ball,e.g., an exploding star or a hadron (fireball) formed after a heavy ion collision [14][15]. In such a case we are interested in an equation of state of such an object. Applying the formula (60) and the relation between $\tilde{E}$ and $\tilde{\Pi}_{E}(68)$ we can derive the relation between the pressure $\tilde{\Pi}_{E}$, density $n$ and temperature $(\beta a)^{-1}$. For the energy-momentum (82) we obtain

$$
\tilde{\Pi}_{E}=n(\beta a)^{-1}\left(1+c_{0}(1)+4 \tilde{c}_{1}(1)(\beta a)^{-1}\right)\left(1+c_{0}(1)+3 \tilde{c}_{1}(1)(\beta a)^{-1}\right)^{-1} .
$$

Eq.(99) modifies the well-know equilibrium relation $\tilde{\Pi}_{E}=n(\beta a)^{-1}$ true (according to eq.(99)) for low temperatures to $\tilde{\Pi}_{E}=\frac{4}{3} n(\beta a)^{-1}$ at high temperatures. The coefficient in the high temperature limit depends on $N$ in the states (84).

\section{Non-relativistic diffusive fluid}

In sec.5 we have studied an ultrarelativistic diffusion (21) at finite temperature for a hot matter. Then, in sec. 6 the evolution of $\Omega$ continued as an ultrarelativistic diffusion at zero temperature. Finally, in this section we consider Universe evolution in the stage when matter is in the form of macroscopic bodies (we treat it as a dust of heavy particles). We still assume that the Universe evolution is described by the diffusion equation (17) in the limit of a large mass $m$. This is at the same time the non-relativistic limit of eq.(17). The diffusion constant $\kappa$ 
at this stage of evolution is different then the one for the early Universe but we keep the same symbol for it. The diffusive behaviour of a particle (dust) could be related to the gravitational attraction of all other masses in the Universe [7]. In this section we find solutions of the non-relativistic diffusion equation, calculate the corresponding energy momentum tensor $\tilde{T}$, derive the formula for $T_{D}$ and discuss the resulting Einstein equations.

In the non-relativistic limit only the massive terms on the rhs of eq.(17) remain. We redefine the diffusion constant $\kappa^{2} \rightarrow \frac{\kappa^{2}}{2 m}$ in eq.(17) and write the equation in terms of the cosmic time $\partial_{t}=a \partial_{\tau}$. Then, the non-relativistic limit of eq.(17) in the cosmic time reads

$$
\partial_{\tau} \Omega-2 H p \frac{\partial \Omega}{\partial p}=\frac{\kappa^{2}}{2 a^{2}}\left(\frac{\partial^{2} \Omega}{\partial p^{2}}+\frac{2}{p} \frac{\partial \Omega}{\partial p}\right)
$$

where $H=a^{-1} \frac{d a}{d \tau}$ (eq.(18)). We proceed as in sec. 4 where the solution began to evolve from the Jüttner equilibrium distribution. We consider a solution of the diffusion equation starting from the Maxwell distribution at $\tau=\tau_{0}$

$$
\Omega_{0}=n a^{3}\left(\tau_{0}\right)(2 \pi)^{3}\left(2 \pi \kappa^{2}\right)^{-\frac{3}{2}} \theta^{-\frac{3}{2}} \exp \left(-\frac{a^{4}\left(\tau_{0}\right) p^{2}}{2 \theta \kappa^{2}}\right) .
$$

So, $\frac{\theta \kappa^{2}}{a^{4}\left(\tau_{0}\right) m}$ has a meaning of the temperature. The state $\Omega_{0}$ is normalized to the particle density $n$, i.e.,

$$
\sqrt{h}\left(\tau_{0}\right) \int \frac{d \mathbf{p}}{(2 \pi)^{3}} \Omega_{0}=n .
$$

The solution of eq.(100) with the initial condition (101) reads

$$
\Omega_{\tau}=n a^{3}\left(\tau_{0}\right)\left(2 \pi \kappa^{2}\right)^{-\frac{3}{2}}\left(\theta+A_{d}\right)^{-\frac{3}{2}} \exp \left(-\frac{a^{4}(\tau) p^{2}}{2\left(\theta+A_{d}\right) \kappa^{2}}\right)
$$

where

$$
A_{d}(\tau)=\int_{\tau_{0}}^{\tau} d s a^{2}(s)
$$

Note that in contradistinction to $A$ in eq.(28) now the integral in $A_{d}$ is performed over the cosmic time. The energy density in the state (102) is

$$
\begin{aligned}
& \tilde{T}^{00}=\sqrt{h} \int \frac{d \mathbf{p}}{(2 \pi)^{3}} \sqrt{m^{2}+a^{2} p^{2}} \Omega_{\tau} \\
& \simeq \sqrt{h} \int \frac{d \mathbf{p}}{(2 \pi)^{3}}\left(m+\frac{1}{2 m} a^{2} p^{2}\right) \Omega_{\tau}=n m a^{3}\left(\tau_{0}\right) a^{-3}(\tau)+C \kappa^{2}\left(\theta+A_{d}\right) a^{-5}
\end{aligned}
$$

with certain positive constants $C$ (independent of $\kappa$ ). In eq.(104) (as usual) we have cut the non-relativistic approximation of the energy at the second order term. We can now repeat the derivation of the modified Friedmann equation 
(73) with the non-relativistic matter corresponding to $\tilde{\pi}_{E}=0$ in eq.(71) (the "dust") leading to

$$
\frac{3}{8 \pi G}\left(a^{-1} \frac{d a}{d \tau}\right)^{2}=\tilde{T}^{00}-\int_{\tau_{0}}^{\tau} d r a^{-3} \partial_{r}\left(a^{3} \tilde{T}^{00}\right)+\frac{\Lambda}{8 \pi G}\left(\tau_{0}\right) .
$$

Inserting the result (104) for $\tilde{T}^{00}$ we obtain the Friedmann equation

$$
\begin{aligned}
& \frac{3}{8 \pi G}\left(a^{-1} \frac{d a}{d \tau}\right)^{2}=n m a^{3}\left(\tau_{0}\right) a^{-3}(\tau)+C \kappa^{2}\left(A_{d}+\theta\right) a^{-5} \\
& -C \kappa^{2} \int_{\tau_{0}}^{\tau} d r a^{-3}+2 C \kappa^{2} \int_{\tau_{0}}^{\tau} H\left(\theta+A_{d}\right) a^{-5} d r+\frac{\Lambda}{8 \pi G}\left(\tau_{0}\right)
\end{aligned}
$$

Depending on the parameters in eq.(106) it has the asymptotic solution for a large time

$$
a(\tau) \simeq \tau
$$

besides the exponentially growing solutions (77). If there is no diffusion $(\kappa=0)$ then eq.(106) is reduced to the standard Friedmann equation for a cosmological dust.

\section{Summary and outlook}

In our approach we replace the Einstein-Vlasov system investigated by many authors by a dissipative system. The usual form of the $a^{-4}$ dependence of the energy density on the scale factor for an ultrarelativistic fluid can be considered as a result of the Jüttner (or Planck) equilibrium phase space distribution with a time-dependent temperature. It also follows from the energy-momentum conservation of an ideal fluid with the density-pressure relation for massless particles. In this paper we considered phase space distributions resulting from a relativistic diffusion. The diffusion approximation comes out from a general assumption of the Markov property of microscopic interactions. There are some general restrictions on the form of the diffusion resulting from the relativistic invariance and the detailed balance. They are independent of the form of the interaction. The dissipative dynamics neglects some degrees of freedom (in astrophysical context:some forms of matter). For this reason the energy-momentum of the diffusing fluid is not conserved. To some extent we can recover the missing part of the total system from the requirement of the energy-momentum conservation.

We have studied a simplified model of a scenario for the Universe evolution which begins with a diffusion at finite temperature with the Planck or Jüttner equilibrium distribution. At later time (lepton era) a description in terms of an equilibrating diffusion cannot apply. However, assuming that the dynamics remains dissipative we continue with the Markov approximation describing the evolution of the phase space distribution by relativistic diffusion (with a different diffusion constant) without any equilibrium heat bath. The corresponding energy-momentum tensor is not conserved. In order to achieve the conservation law for the total energy-momentum we introduce a "cosmological term". 
Under the assumption of homogeneity of the (dark) matter distribution the cosmological term is determined by the form of the energy-momentum of the diffusing fluid. We have assumed that particles are massless. This assumption can be justified at an early stage of Universe evolution either as an ultrarelativistic approximation or referring to the grand unified theory in which masses arise at lower temperatures through the symmetry breaking. At the later stage the massless fluid must be considered as a mathematical simplification (21) of eq.(17) which allows to solve the diffusion equation in order to derive the form of the energy-momentum tensor as a function of the scale factor. Inserting the solution of the diffusion equation into the Einstein equations we can derive a dissipative version of the Friedmann equation. We have studied some consequences of this modification of the Friedmann equation. We show that the cosmological term starting from the cosmological constant (as a free integration parameter) is decreasing with time. If it tends to zero then the Friedmann equation has a solution of the form $a \simeq \tau$ for a large time. If the cosmological constant is large enough then the Friedmann equation has an exponentially growing solution. We would need to apply numerical methods to study the scale factor evolution in order to see whether it can support a fast expansion at an intermediate time simulating the inflation. At the later stage of Universe evolution when matter acquires a macroscopic form we describe the evolution by a non-relativistic diffusion of a dust of heavy particles. We show that such an evolution leads to the same conclusions concerning the long time behaviour as the relativistic diffusion at zero temperature. We have proposed a simplified model of the Universe evolution which has a built in equilibrium stage and allows explicit solutions. The approach could be considered as an approximation of the collision term in the Boltzmann equation (treated, e.g., in [13]) by a diffusion applied to the case when some scattering processes causing the energy dissipation remain unknown. It gives a phenomenological description of quantum processes in an early Universe. We were able to study some consequences of our assumptions. In particular, it could be seen that the dissipation determines the large time behaviour of $a(\tau)$. The early times of the Universe dynamics are accessible to astronomical observations through their impact on the structure formation. We intend to continue the study by an admission of a non-homogeneity of the metric in order to investigate the result of the diffusive dynamics of the hot Universe on the propagation of disturbances. For a small time the modified Jüttner solutions of the diffusion equations at finite temperature lead to a slower approach to the $a=0$ singularity of the scale factor. Such a behaviour could possibly prevent the appearance of the singularity at $\tau=0$ in a more general dissipative dynamics. The admittance of polynomial modifications of the Jüttner distribution (as solutions of the diffusion equation) leads to a modification of the equation of state defined as a relation between the density, temperature and pressure.

The acknowledgements

The author thanks an anonymous referee for inspiring questions. Interesting discussions with Andrzej Borowiec and Marek Szydlowski are gratefully ac- 
knowledged. The research is supported by Polish NCN grant No.2013/09/B/ST2/03455.

\section{References}

[1] J. Ehlers,P. Geren and R.K. Sachs, J.Math.Phys.9,1344(1968)

[2] D. Boyanowsky, H.J. de Vega and N.G. Sanchez, Phys.Rev. D77,043518(2008)

[3] V. I. Pavlov and E.P. Tito, Phys.Rev. D85,103010(2012)

[4] H. Andreasson, Living Rev.Relativity, 14,4(2011);arXiv:gr-qc/1106.1367

[5] E. M. Lifshits and L.P. Pitaewskij, Physical Kinetics, Pergamon Press,1981

[6] S. Chandrasekhar, Rev.Mod.Phys.15,1(1943)

[7] S. Chandrasekhar, Ap.J.97,255(1943)

[8] R. Wielen, Astron.Astroph.60,263(1977)

[9] J. Binney and S. Tremaine, Galactic Dynamics,2nd ed.,Princeton,2008

[10] C-P. Ma and E. Bertschinger, Ap.J.612,28(2004),

E. Bertschinger, arXiv:astro-ph/0608583

[11] F. Ferrer and D.R. Hunter, arXiv:astro-ph/1306.658

[12] A. Albrecht and P.J. Steinhardt, Phys.Rev.Lett.48,1220(1982)

[13] S. Dodelson, Modern Cosmology, Academic Press, New York, 2003

[14] J. Rafelski, arXiv:astro-ph/1306.2471

[15] U. Heinz, arXiv:nucl-th/1304.3634

[16] Z. Haba,Journ.Phys.A46,155001(2013)

[17] Z.Haba,Mod.Phys.Lett.A28,1350091(2013)

[18] G.Schay,PhD thesis,Princeton University,1961

[19] R.Dudley, Arkiv for Matematik,6,241(1965)

[20] F. Jüttner, Ann.Phys.(Leipzig)34,856(1911)

[21] Z. Haba, Phys.Rev.E79,021128(2009)

[22] J.A. Alcantara and S.Calogero, Kin.Rel. Mod.4,401(2011) 
[23] J. Dunkel and P. Hänggi, Phys.Rev.E72,036106(2005)

[24] C. Chevalier and F. Debbasch, J.Math.Phys.49,043303(2008)

[25] Z. Haba, Physica,A390,2776(2011)

[26] A.S. Kompaneets, Sov.Phys.JETP 4,730(1957)

[27] S. Weinberg, Cosmology,Oxford Univ. Press,2008

[28] G. Chacon-Acosta and G.M. Kremer, Phys.Rev.E76, 021201(2007)

[29] Z.Haba,Mod.Phys.Lett.A25,2681(2010)

[30] Z. Haba, Class.Quant.Grav.27,095021(2010)

[31] A. A. Penzias and R.W. Wilson, Ap.J.142.419(1965)

R.H. Dicke, P.J.E. Peebles, P.G. Roll and D.T. Wilkinson, Ap.J.142,414(1965)

[32] S. Calogero, JCAP,11,016(2011)

[33] S. Calogero, Journ.Geom.Phys.62,2208(2012)

[34] S. Weinberg, Rev.Mod.Phys. 61,1(1988)

[35] G.F.R. Ellis, H. van Elst,J Murugan and J-P. Uzan, Class.Quant.Grav.28,225007(2011)

[36] S.M. Carroll, arXiv:astro-ph/0310342

[37] N. Straumann, arXiv:astro-ph/0203330

[38] S.W. Hawking and G.F.R. Ellis, The Large Scale Structure of Space-Time, Cambridge Univ. Press, 1973

[39] C. Cercignani and G.M. Kremer, The Relativistic Boltzmann Equation: Theory and Applications, Birkhäuser, Basel, 2002

[40] C. Chevalier and F. Debbasch, J. Math.Phys.48,023304(2007)

[41] R. Durrer, The Cosmic Microwave Background, Cambridge,2008

[42] J. Franchi and Y.Le Jan, Comm.Pure Appl.Math.60,187(2007)

[43] J. Bernstein, Kinetic Theory in the Expanding Universe,Cambridge,1988

[44] J.M. Overduin and F.I. Cooperstock, Phys.Rev. D58,043506(1998) 
[45] M. Kunik, S. Qamar and G. Warnecke, Numer.Math.97,159(2004)

[46] S.M. Carrol, M. Hoffman and M. Troden, Phys.Rev.D68,023509(2003)

[47] T-P Cheng, Relativity, Gravitation and Cosmology, 2nd ed.,Oxford, 2010

[48] J.L. Lopez and D.V. Nanopoulos, arXiv:hep-ph/9501293

[49] F. Melia and A.S.H. Shevchuk,MNRAS., 419,2579(21012); arXiv:astro$\mathrm{ph} / 1109.5189$ 